\title{
From Big Smartphone Data to Worldwide Research: The Mobile Data Challenge
}

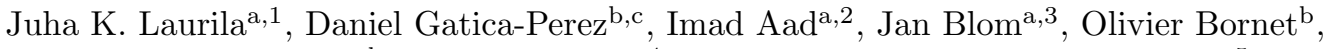
Trinh Minh Tri Dob ${ }^{\mathrm{b}}$ Olivier Dousse ${ }^{\mathrm{a}, 4}$, Julien Eberle ${ }^{\mathrm{a}, \mathrm{c}}$, Markus Miettinen ${ }^{\mathrm{a}, 5}$

\author{
${ }^{a}$ Nokia Research Center, Lausanne, Switzerland \\ ${ }^{b}$ Idiap Research Institute, Martigny, Switzerland \\ ${ }^{c}$ EPFL, Lausanne, Switzerland
}

\begin{abstract}
This paper presents an overview of the Mobile Data Challenge (MDC), a large-scale research initiative aimed at generating innovations around smartphone-based research, as well as community-based evaluation of mobile data analysis methodologies. First, we review the Lausanne Data Collection Campaign (LDCC) - an initiative to collect unique, longitudinal smartphone data set for the MDC. Then, we introduce the Open and Dedicated Tracks of the MDC; describe the specific data sets used in each of them; discuss the key design and implementation aspects introduced in order to generate privacypreserving and scientifically relevant mobile data resources for wider use by the research community; and summarize the main research trends found among the $100+$ challenge submissions. We finalize by discussing the main lessons learned from the participation of several hundred researchers worldwide in the MDC Tracks.
\end{abstract}

Keywords:

mobile data challenge, smartphone data collection, human behavior analysis

\section{Introduction}

Mobile phone technology has transformed the way we live, as phone adoption has increased rapidly across the globe [1]. This has widespread social implications. The phones themselves have become instruments for fast communication and collective participation. Further, different user groups are using them in creative ways. At the same

\footnotetext{
Paper submitted to the Mobile Data Challenge Special Issue.

Email addresses: juha.k.laurila@nokia.com (Juha K. Laurila), gatica@idiap.ch (Daniel Gatica-Perez), aad@iam.unibe.ch (Imad Aad), janblom@google.com (Jan Blom), bornet@idiap.ch (Olivier Bornet), do@idiap.ch (Trinh Minh Tri Do), olivier.dousse@here.com (Olivier Dousse), julien.eberle@epfl.ch (Julien Eberle), markus.miettinen@trust.cased.de (Markus Miettinen)

${ }^{1}$ J. K. Laurila is currently affiliated with Nokia Research Center, Helsinki, Finland.

${ }^{2}$ I. Aad is currently affiliated with University of Bern, Switzerland.

${ }^{3} \mathrm{~J}$. Blom is currently affiliated with Google Zurich, Switzerland.

${ }^{4} \mathrm{O}$. Dousse is currently affiliated with HERE, Berlin, Germany.

${ }^{5} \mathrm{M}$. Miettinen is currently affiliated with CASED, Darmstadt, Germany.

Preprint submitted to Pervasive and Mobile Computing
} 
time, the number of sensors embedded in phones and the applications built around them have exploded. In the past few years, smartphones started to carry sensors like GPS, accelerometer, gyroscope, microphone, camera and Bluetooth. Related applications and services cover, for example, information search, entertainment, and healthcare.

The ubiquity of mobile phones and the increasing wealth of the data generated from sensors and applications are giving rise to a new research domain across computing and social science. Researchers are beginning to examine issues in behavioral and social science from the Big Data perspective - by using large-scale mobile data as input to characterize and understand real-life phenomena, including individual traits, as well as human mobility, communication, and interaction patterns $[2,3,4]$.

This research, whose findings are important to society at large, has been often conducted within corporations that historically have data sets, including telecom operators [5] or Internet companies [6], or through granted data access to academics in highly restricted forms [3]. Some initiatives, like [7], have collected publicly available data sets, which are to some extent limited in scope. Clearly, government and corporate regulations for privacy and data protection play a fundamental and necessary role in protecting all sensitive aspects of mobile data. From the research perspective, this also implies that mobile data resources are scarce and often not ecologically valid to test scientific hypotheses related to real-life behavior. By ecologically valid data, we mean everyday life data from users who actually use the sensing phone as their personal primary device.

The Mobile Data Challenge (MDC) by Nokia was motivated by our belief in the value of mobile computing research for the common good - i.e., of research that can result in deeper scientific understanding of human phenomena, advanced mobile experiences, and technological innovations. Guided by this principle, in January 2009 Nokia Research Center Lausanne (NRC), Idiap Research Institute, and EPFL started an initiative to create large-scale mobile data research resources. This included the design and implementation of the Lausanne Data Collection Campaign (LDCC), an effort to collect a longitudinal smartphone data set from nearly 200 volunteers in the Lake Geneva region over 18 months. It also involved the definition of a number of research tasks with clearly specified experimental protocols. From the very beginning, the intention was to share these resources with the research community. This required the integration of holistic and proactive approaches on privacy according to the privacy-by-design principles $[8,9]$.

The MDC was the visible outcome of nearly three years of work in this direction. The Challenge provided researchers with an opportunity to analyze a relatively unexplored data set including rich mobility, communication, and interaction information. The MDC comprised of two alternatives through an Open Research Track and a Dedicated Research Track. In the Open Track, researchers were given the opportunity to approach the data set from an exploratory perspective, by proposing their own tasks according to their interests and background. The Dedicated Track gave researchers the possibility to take on up to three tasks to solve, related to prediction of mobility patterns, recognition of place categories, and estimation of demographic attributes. Each of these tasks had properly defined experimental protocols and standard evaluation measures to assess and rank all contributions.

This paper presents a description and analysis of the Mobile Data Challenge 2012. The paper is an extended version of the MDC overview paper originally presented in $[10,11]$. In this paper, we expand our analysis of the submissions and the corresponding results, and reflect on the MDC process and its outcomes. The paper is organized as 
follows. Section 3 summarizes the LDCC data. Section 4 introduces the MDC tracks and tasks. Section 5 provides details on the specific data sets used for the MDC. Section 6 summarizes the MDC process schedule. With the goal of presenting a global understanding of the participation of the research community in the Challenge, Section 7 presents a brief analysis of the MDC contributed papers. On one hand, we summarize the main research questions tackled by the papers contributed to the Open Track; on the other one, we present a comparative analysis of the objective performance obtained by the contributions in the Dedicated Track. A selection of these papers, in significantly extended versions, conforms the current Special Issue. In Section 8, we reflect upon the MDC process and discussed the main lessons we learned along the way. We hope that these experiences can inform the design and implementation of future initiatives. Finally, Section 9 contains some final remarks.

\section{Related work}

This section presents an overview of mobile phone datasets used in previous studies. We divide them in two categories: mobile network operator (MNO) data and smartphone sensing data.

Call Detail Records (CDRs) are the main source of MNO data, and are collected for billing and network traffic monitoring. In today's mobile networks, each CDR may correspond to a voice call, video call, SMS, or other operator data services. Besides basic statistics of a call event (e.g., caller ID, callee ID, time, duration), the ID of the cell tower that the phone connects to is also available. As cell tower locations are known by MNOs, their IDs indirectly provide the location traces of users with a coarse resolution. MNO data are then useful for analyzing not only communication patterns but also human mobility. As an example, Gonzalez et al. analyzed individual human mobility patterns from a proprietary dataset provided by a European MNO having roughly 6 million customers [3]. The analysis was done on a subset of 100,000 anonymous users over 6 months, showing that several fundamental properties of individual human mobility can be captured. A similar dataset, collected from hundreds of thousands of people in the US, was used by AT\&T researchers to characterize human mobility patterns with a particular focus on practical applications, such as estimating carbon emissions [12]. In both datasets, privacy was carefully handled by anonymization and also by limiting the studies to report aggregate results only.

Researchers worldwide had the opportunity to analyze MNO data via the Data for Development (D4D) challenge, which was launched in late 2012 by Orange [13]. To get access to the dataset generated by about 5 million users over 5 months in Ivory Coast, each research team had to submit a short description of their openly defined research project, which was then reviewed by the organizers. Instead of giving raw CDR data, Orange provided four types of preprocessed data: location traces of 50,000 users with native resolution (cell IDs), location traces of 500,000 users with coarse resolution (cell IDs were replaced by sub-prefecture IDs), hourly antenna-to-antenna communication traffic, and ego communication subgraphs of 5,000 randomly chosen users. The selected and winning projects were presented in May 2013.

Targeting the machine learning and data mining communities, the KDD cup 2009 was another example of MNO data [14]. Based on a large marketing database of customers, the goal was to predict the propensity of customer to switch provider, buy new product 
or services, or buy upgrades or add-ons proposed to them. The dataset consisted of precomputed attributes of customers, provided as a 100,000 $\times 15,000$ customer-attribute matrix, and the target values for the training set. In the dedicated tasks of MDC, we also predefined specific prediction problems, but participants were free to exploit the raw sensor data for their prediction methods.

Regarding smartphone data, the large number of sensors and the possibility to run customized recording software have made smartphones a great option to collect data in an unprecedented quantity and granularity. Smartphone sensing has shown to be effective across multiple scales: individuals [15], groups of people with shared interests (e.g., the Garbage Watch project [16]), and also at community/country scale (e.g., Participatory Urbanism [17]). While energy remains the bottleneck for mobile devices, energy-efficient sensing techniques have opened the possibility of continuous sensing in daily life [18, 19]. Smartphone data is currently being collected and analyzed by academic researchers, small companies, and large corporations. For example, the CitySense consumer application (developed by SenseNetworks) shows human hotspots in a city in real-time by combining online data with billions of GPS points generated over a few years [20]. Mobile Millennium is a traffic-monitoring system that accumulates traffic information from mobile users and then broadcasts highway and arterial information in real-time [21]. This mobile application was downloaded by more than 5000 users during one year. In another system to estimate travel time, Thiagarajan et al. [22] collected a dataset of GPS and WiFi location samples from nearly 800 hours of actual commuter drives, gathered with iPhones and embedded in-car computers.

While smartphone sensing is very appealing, the available data open to the research community is very limited. The Reality Mining project pioneered this direction by releasing a dataset recorded with Nokia 6600 smartphones by 100 students over 9 months at MIT [23]. The data included call logs, Bluetooth devices in proximity, cell tower IDs and application usage. Inspired by the open-access idea of Reality Mining, the LDCC was launched in Switzerland in 2009 and benefited from the lessons learned about the design of Reality Mining. Compared to Reality Mining, the population of LDCC is more diverse, with a mixture of students and professionals; the LDCC was also twice as long (18 months). Notably, the LDCC also included additional data types that are highly relevant such as GPS, WLAN, and accelerometer. Additionally, the LDCC data was collected in Europe, and therefore it reflects the lifestyle of a European population.

Finally, in another effort to create large mobile data for research on activity recognition, the HASC project created a shared corpus. The organizers provided the recording software and ask participants (mainly researchers who want to use the data) to contribute their own data to the corpus [24]. In 2012, they reported to have data from 24 teams, 136 subjects, and 4 data types: accelerometer, gyroscope, GPS, and magnetic field sensor. Note that this corpus consists of monitored (and labeled) records only, as opposed to LDCC in which data samples were recorded automatically in the phone's background.

\section{The Lausanne Data Collection Campaign (LDCC)}

The LDCC aimed at designing and implementing a large-scale campaign to collect smartphone data in everyday life conditions, grounding the study on a European culture. The overall goal was to collect quasi-continuous measurements covering all sensory and other available information on a smartphone. In this way, we were able to capture 
phone users' daily activities unobtrusively, in a setting that implemented the privacy-bydesign principles $[8,9]$. The collected data included a significant amount of behavioral information, including both individual and relational aspects. The intention was to enable the investigation of a large number of research questions related to personal and social context, including mobility, phone usage, communication, and interaction. All content, like image files and text messages, was excluded from recording as it was considered too sensitive. Instead, log-files with metadata were collected both for imaging and messaging applications. This section provides a summary on the LDCC implementation and captured data types. An initial paper introducing LDCC and its data types and statistics appeared in [25]. Part of the material in this section has been adapted from it.

\section{1. $L D C C$ design}

Nokia Research Center, Idiap, and EPFL partnered towards the LDCC since January 2009. After the implementation and evaluation of the sensing architecture, and the recruitment of the initial pool of volunteers, the data collection started in October 2009. Over time, smartphones with data collection software were allocated to close to 200 volunteers in the Lake Geneva region. A viral approach was used to promote the campaign and recruit volunteers. This resulted in a population with social connections to other participants, as well as greater variation in terms of demographic attributes compared to previous initiatives [23]. This was a consequence of the fact that the participants were guided to recruit further campaign members representing different social connections (like family member, colleague, neighbour, hobby mate, etc.) A key aspect of the success of the LDCC was the enthusiastic participation of volunteers who agreed to take part in the campaign and share their data mainly driven by selfless interest. The campaign concluded in March 2011.

Data was collected using Nokia N95 phones and a client-server architecture that made the collection process invisible to the participants. A seamless implementation of the data recording process was key to make a longitudinal study feasible in practice - many participants remained in the study for over a year. Another important target for the client software design was to reach an appropriate trade-off between quality of the collected data and phone energy consumption.

The collected data was first stored in the device and then uploaded automatically to a Simple Context server via WLAN. The server received the data, and built a database that could be accessed by the campaign participants. The Nokia Simple Context backend had been developed earlier by the Nokia Research Center in Palo Alto. Additionally, a data visualization tool was developed to offer a "life diary" type of view for the campaign participants on their data. Simultaneously, an anonymized database was populated, from which researchers were able to access the data for their purposes. Fig. 1 presents a block diagram of the data collection architecture.

\subsection{Data characteristics}

The LDCC initiative produced a unique data set in terms of scale, temporal dimension, and variety of data types. The campaign population reached 185 participants. Basic demographics showed a bias towards male participation ( $62 \%$ male, $38 \%$ female), and concentration on young individuals (the age range 22-33 year-old accounts for roughly two thirds of the population.) Clearly, the LDCC population is not a fair random sample of 


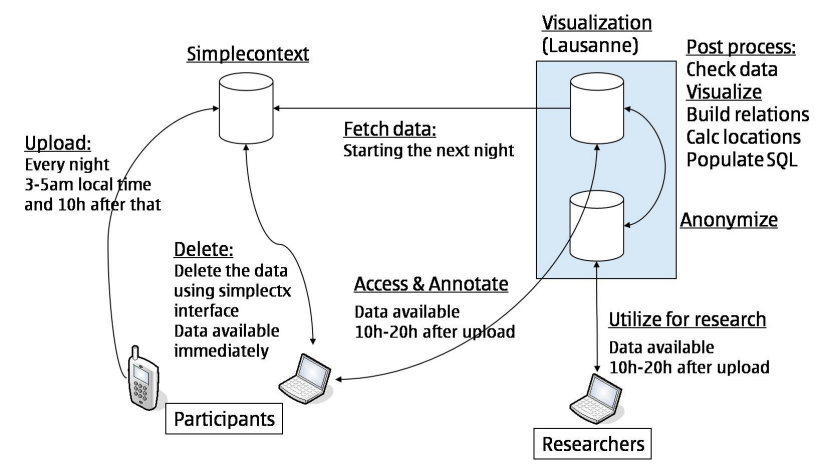

Figure 1: LDCC data flow, progressing from mobile data from volunteers to anonymized data for research $[25])$.

the general population in French-speaking Switzerland. That said, the LDCC population is diverse in terms of demographic attributes when compared to previous initiatives [23], where essentially all volunteers were related to a university community. In LDCC, there is a proportion of users who do not have connections to the local universities, and who have other professions and age ranges. This diversity gave the possibility of designing and implementing a task about predicting demographic attributes as part of MDC.

A bird-eye's view on the LDCC in terms of data types is shown in Table 1. As can be seen, data types related to location (GPS, WLAN), motion (accelerometer), proximity (Bluetooth), communication (phone call and SMS logs), multimedia (camera, media player), application usage (user-downloaded applications in addition to system ones), and audio environment (optional) were recorded. The numbers themselves reflect a combination of experimental design choices (e.g., every user had the same phone) and specific lifestyle choices (e.g., many participants use public transportation).

Due to space limitations, it is not possible to visualize multiple data types here. A compelling example, however, is presented in Fig. 2, which plots the raw location data of the LDCC on the map of Switzerland for the volunteer population after 1 week, and then after $1,3,6,12$, and 18 campaign months. When considered in detail, the geographical coverage of the LDCC allows a reasonable tracing of the main routes on the map of Suisse Romande - the French-speaking, western part of Switzerland - and partially also of other regions of the country.

In addition to contributing phone data, LDCC participants also agreed to fill a small number of surveys during the data recording process. Two types of survey data were important for the later development of the MDC: (1) a set of manual semantic labels for frequently and infrequently visited places for each user, and (2) basic demographic attributes. The relevant places for each user were first detected automatically with a method discussed in [26]. After that, the campaign participants specified place categories from a fixed list of tags (home, work, leisure places, etc.). With respect to demographics, participants self-reported their attributes like gender, age group, marital status, job type, etc. 


\begin{tabular}{|l|r|}
\hline Data type & Quantity \\
\hline \hline Calls (in/out/missed) & 240,227 \\
SMS (in/out/failed/pending) & 175,832 \\
Photos & 37,151 \\
Videos & 2,940 \\
Application events & $8,096,870$ \\
Calendar entries & 13,792 \\
Phone book entries & 45,928 \\
Location points & $26,152,673$ \\
Unique cell towers & 99,166 \\
Accelerometer samples & $1,273,333$ \\
Bluetooth observations & $38,259,550$ \\
Unique Bluetooth devices & 498,593 \\
WLAN observations & $31,013,270$ \\
Unique WLAN access points & 560,441 \\
Audio samples & 595,895 \\
\hline
\end{tabular}

Table 1: LDCC main data types and amount of data.
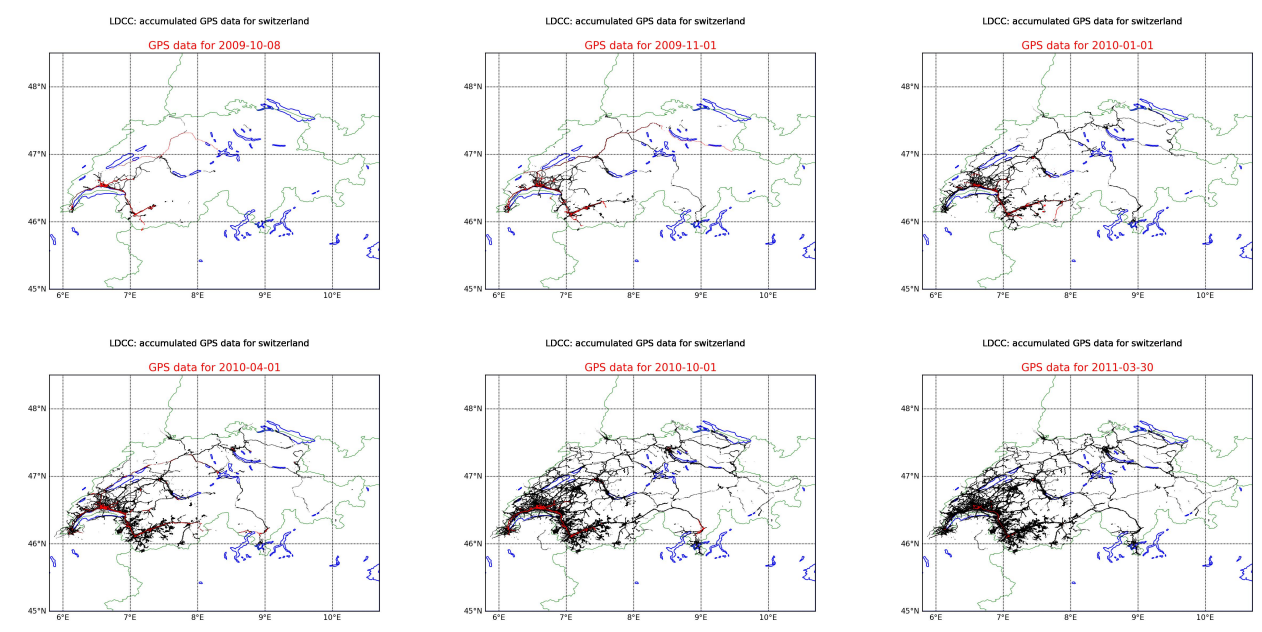

Figure 2: LDCC location data (in black) plotted at the country level (outlined in green) after 1 week, 1 month, 3 months, 6 months, 12 months, and 18 months of campaign. The data for each specific day is plotted in red. 


\subsection{Privacy}

Privacy played an essential role in the design and implementation of the LDCC, given the nature and scale of the data shared by the participants. In order to satisfy the ethical and legal requirements to collect data while protecting the privacy of the participants, the LDCC team implemented an approach based on multiple mechanisms complementing each other. The approach can be summarized as follows (more details can be found in [25]):

1. Communication with volunteers about privacy. Following Nokia's general privacy policy, we obtained written consent from each individual participating in the LDCC. We explicitly stated that data would be collected for research purposes. All participants were informed about their data rights, including the right to access their own collected data and to decide what to do with it (e.g. to delete data entries if they opted to do so). The participants had also the opportunity to opt-out at any moment.

2. Data security. The data was recorded and stored using the best industry practices in this domain.

3. Data anonymization. By design, the LDCC did not store any content information (e.g. photo files or text messages). The major portion of the collected data consisted of event logs, and when sensitive data beyond logs was collected, it was anonymized using state-of-the-art techniques and/or aggregated for research purposes [9]. Examples include the use of pseudonyms instead of identifiable data, and the reduction of location accuracy around potentially sensitive locations. Apart from the core team directly involved with the data collection/anonymization procedures, any other researchers were only granted access to the anonymized data.

4. Commitment of researchers to respect privacy. Privacy protection of the LDCC data purely by automatic anonymization techniques is not possible so that the data value for research is simultaneously maintained. On one hand, technological approaches for privacy are not $100 \%$ effective for all data types, as mobile privacy research has often shown. On the other hand, the degree of degradation of some data types (e.g. location accuracy) can make the data very limited in practice to investigate certain research hypotheses. Several measures were thus needed in order to maximize the value for research of the data. Regarding privacy, in addition to technical means, agreementbased countermeasures were necessary. A first set of trusted researchers was able to work with the LDCC data after agreeing in written form to respect the anonymity and privacy of the volunteering LDCC participants. This practically limited the access to the LDCC data to a small number of authorized partners. After this initial data sharing experience, the next step was to outreach the mobile computing community at large, which motivated the creation of the MDC, discussed in detail in the next sections.

\section{Overview of MDC tracks}

The MDC proposed two alternatives through an Open Research Track and a Dedicated Research Track. In the Open Track, researchers were given the opportunity to approach the data set from an exploratory perspective, by proposing their own tasks according to their interests and background. The Dedicated Track gave researchers the possibility to take on up to three tasks to solve, related with prediction of mobility patterns, recognition of place categories, and estimation of demographic attributes. 
MDC's original intention was to be inclusive at a global scale. Other previous successful evaluation initiatives in computing, like those organized by NIST in several areas [27, 28] or the Netflix challenge [29, 30] focused on either one or at most a small number of tasks with objective evaluation protocols. This was also a guiding principle for the MDC. On the other hand, the nature of mobile data is highly exploratory, so there was a clear benefit in encouraging and welcoming novel ideas.

Learning from these past experiences, we decided that MDC would feature both open and pre-defined options to participate. With this idea in mind we created two tracks. The Open Track was designed to receive ideas directly proposed by the community. On the other hand, a set of challenges was given in the Dedicated Track, which defined three classification/prediction tasks. These tasks covered different aspects related to the characterization of mobile users and places. The Tracks targeted researchers with different profiles.

\subsection{The Open Track}

This Track allowed participants to propose their own Challenge task based on their interests and background. Examples proposed to the participants included the discovery of behavioral patterns through statistical techniques, the development of efficient mobile data management methods, or the design of ways to visualize mobile Big Data.

\subsection{The Dedicated Track}

This Track gave the possibility of taking up to three concrete tasks, with properly defined training and test sets, and evaluation measures used to assess and rank all the contributions. The participants of the Dedicated Track were allowed to define their own features and algorithms. The three tasks of this Track followed a two-stage schedule. In the first stage, the training set (including raw data, labels, and performance measures) was made available to the participants, who were expected to design their features and train their models using this data set. In the second stage, the test set was made available, except for the labels which were kept hidden. Participants were allowed to submit up to five runs of results, and the evaluation of all methods was conducted by the MDC organizers.

\subsubsection{Task 1: Semantic Place Prediction}

Inferring the meaning of the most significant places that a user visits is an important problem in mobile computing [5]. This has been an issue that, under slightly different formulation, has been studied by a significant amount of literature. The semantic labels attributed to places have typically included basic categories common to a population (home, work, restaurant) but could also be personalized (e.g. differentiating the main and second homes of a user). The goal of the task was to predict the semantic meaning of these places for a number of users of the MDC data. Each place was represented by a history of visits over a period of time, for which other contextual information sensed by the user's smartphone was available. On one hand, participants had to extract relevant features for predicting these semantic labels. On the other hand, specific methods for this task had to be developed, given the particular type of input information (sequences of visits as opposed to geographic location). Importantly, it was decided that geo-location would not be provided as a feature for this task for privacy reasons, as some of the place 
categories are privacy-sensitive (like home and work.) Several other types of phone data were provided as features (see next Section). Semantic place labels (manually provided by the LDCC users through surveys) were given as part of the MDC training set.

\subsubsection{Task 2: Next Place Prediction}

Predicting the location of phone users has relevance for context-aware and mobile recommendation systems [31]. This topic has been increasingly addressed in the literature under different definitions of the prediction task, including predicting the next location given the current one; predicting a set of locations likely to be visited in a future time interval; and predicting the duration of future visits. The goal of the MDC task was to predict the next destination of a user given the current context, by building user-specific models that learn from user mobility history, and then applying these models to the current context to predict where the users go next. In the training phase, the mobility history of each user was represented by a sequence of visits to specific places, and several types of phone data associated with these visits were made available. Furthermore, in the testing phase, previously unseen data from the same set of users was provided, with the goal of predicting the next place for each user given their current place and a short history of places.

\subsubsection{Task 3: Demographic Attribute Prediction}

The knowledge of basic demographic attributes is an important aspect in user modeling that can find diverse applications, ranging from churn prediction in the context of mobile operator preferences to informing social science studies, where phone data could complement traditional survey-based methods [32]. The goal of the task proposed in MDC was to infer basic demographic groups of users based on behavioral information collected from the phones. As discussed earlier, some of the voluntarily-provided demographic information in the LDCC included self-reported gender, age group, marital status, job type, and number of people in the household. This information was provided for training and kept hidden for testing. Three subtasks, namely gender, marital status, and job prediction were formulated as classification problems, for which classification accuracy was used as evaluation measure. The two remaining attributes corresponded to regression problems, for which the root mean square error (RMSE) was used as evaluation measure. Each subtask contributed equally to the final score which was defined as the average of relative improvements over baseline performance.

\section{MDC data}

This section presents an overview of the MDC dataset and the corresponding preparation procedures. We first describe the division of the original LDCC data that was needed to address the different MDC tasks. We then summarize the data types that were made available. We conclude by discussing the procedures related to privacy and data security.

\subsection{Division of the dataset}

The datasets provided to the participants of the MDC consisted of slices of the full LDCC dataset. Slicing the data was needed to create separate training and test sets for 
the tasks in the Dedicated Track, but was also useful to assign the richest and cleanest parts of the LDCC dataset to the right type of challenge. Four data slices were created:

Set A: Common training set for the three dedicated tasks.

Set B: Test set for demographic attribute and semantic place label prediction tasks.

Set $C$ : Test set for location prediction task.

Open set. Set for all open track entries.

The overall structure of the datasets is given in Figure 3. The rationale behind this structure was the following. First, the participants of the LDCC were separated in three groups, according to the quality of their data with respect to different criteria. The 80 users with the highest-quality location traces were assigned to sets A and C. Set A contains the full data for these users except the 50 last days of traces, whereas set $\mathrm{C}$ contains the 50 last days for which location data is available for testing.

In order to maximize the use of our available data, we reused Set $\mathrm{A}$ as a training set for the other two dedicated tasks. A set of 34 further users was selected as a test set for these tasks and appeared as Set B. In this way, models trained on the users of Set A can be applied to the users of their most visited locations.

Demographic data and semantic labels, as explained in Section 3, were collected through surveys. Since all steps of the LDCC participation were fully voluntary, a number of users chose not to complete the surveys, or filled them only partially. Therefore, the participants for whom complete questionnaire data was not available were assigned to the last set, which was used for the Open Track. In total, 38 users were assigned to this dataset. Overall, with this data split, a total of 152 LDCC participants were included in the MDC datasets.

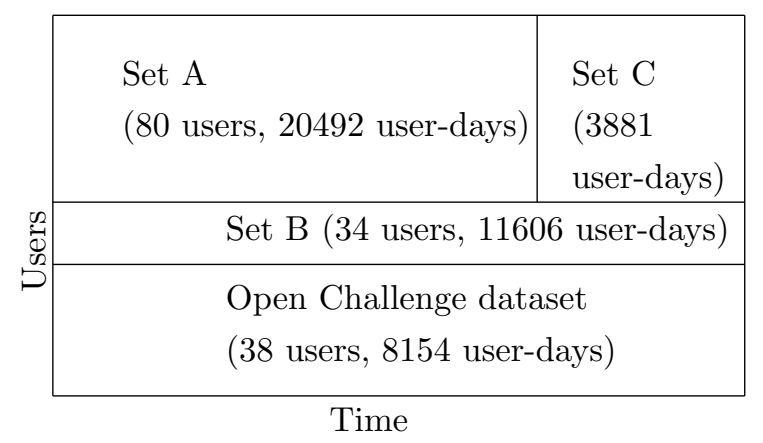

Figure 3: Division of the MDC dataset into four challenge subsets. For each set, the total number of user-days with data is also shown. 


\subsection{Data types}

For both Open and Dedicated Tracks, most data types were released in a raw format except a few data types that had to be anonymized. There are two main differences between the Open Track data and the Dedicated Track data. First, the physical location (based on GPS coordinates) was available in the Open Track but not in the Dedicated Track. We released a preprocessed version of the location data in the form of sequences of visited places for the Dedicated Track. This allowed to study performance of algorithms in a location privacy-preserving manner. The second main difference was the availability of relational data between users. This included both direct contacts (e.g., when a user calls another user) and indirect contacts (e.g., if two users observe the same WLAN access point at the same time then they are in proximity). We decided to keep this data in the Open Track but removed it in the Dedicated Track since it could have potentially revealed the ground truth to be predicted. In the anonymization algorithm, a common hashing key was used for the users selected for the Open Track data sets. On the other hand, we used a different hashing key for each user in the Dedicated Track.

Common data types. A table is associated to each data type in which each row represents a record such as a phone call or an observation of a WLAN access point. User IDs and timestamps are the basic information for each record. Specific information of each data type is detailed in Table 2 .

Data types for Open Track only. Geo-location information was only available in the Open Track. In addition to GPS data, we also used WLAN data for inferring user location. The location of WLAN access points was computed by matching WLAN traces with GPS traces during the data collection campaign. The description of geo-location data is reported in Table 3.

Location data in Dedicated Track. Physical location was not disclosed in the Dedicated Track. For each user in the dedicated track, the raw location data (based on GPS and WLAN) was transformed into a symbolic space which captures most of the mobility information and excludes actual geographic coordinates. This was done by first detecting visited places and then mapping the sequence of coordinates into the corresponding sequence of place visits (represented by a place ID). A place was defined as a small circular region with 100-meter radius that had been visited for a significant amount of time. The place discovery process was done with a two-step approach [26]. First, the sequence of coordinates was segmented into stay points and transitions, where stay point was defined as a subsequence of the location trace for which the user stayed within a small circular region (radius=100 meters) for at least 10 minutes. In the second step, the detected stay points were grouped by a grid clustering algorithm which is based on a uniform grid where each cell is a square region of side length equal to 30 meters. The algorithm starts with all stay points in the working set and an empty set for stay regions. At each iteration, the algorithm looks for the $5 \times 5$-cell region that covers most stay points and removes the covered stay points from the working set. This process is repeated until the working set is empty. Finally, the centers of $5 \times 5$-cell regions are used to define circular stay regions that we called places. Note that the place extraction was done for each user separately, therefore places are user-specific. We also ordered places by the time of the first visit (thus, the visit sequence starts with place ID=1). Although the absolute coordinates of places were not provided, a coarse distance matrix between places was computed for each user and provided for the MDC participants of this track. 


\begin{tabular}{|c|c|}
\hline data $t$ & escription \\
\hline $\begin{array}{l}\text { accel.csv } \\
\text { application.csv }\end{array}$ & $\begin{array}{l}\text { user ID, time, motion measure, and accelerometer samples. } \\
\text { user ID, time, event, unique identifier of the application, } \\
\text { and name of the application. }\end{array}$ \\
\hline bluetooth.csv & $\begin{array}{l}\text { user ID, time, first } 3 \text { bytes of MAC address, anonymized } \\
\text { MAC address, anonymized name of the Bluetooth device. }\end{array}$ \\
\hline calendar.csv & $\begin{array}{l}\text { user ID, time, entry ID, status (tentative/confirmed), entry } \\
\text { start time, anonymized title, anonymized location, entry } \\
\text { type (appointment/event), entry class (public/private), last } \\
\text { modification time of the entry. }\end{array}$ \\
\hline callog.csv & $\begin{array}{l}\text { user ID, call time, call type (voice call/show message), SMS } \\
\text { status (delivered, failed, etc.), direction (incoming, outgo- } \\
\text { ing, missed call), international and region prefix of phone } \\
\text { number, anonymized phone number, indicator if number is } \\
\text { in phone book, call duration. }\end{array}$ \\
\hline contacts.csv & $\begin{array}{l}\text { user ID, creation time, anonymized name, international and } \\
\text { region prefix of phone number, last modification time. }\end{array}$ \\
\hline gsm.csv & $\begin{array}{l}\text { user ID, time, country code and network code, anonymized } \\
\text { cell id, anonymized location area code, signal strength. }\end{array}$ \\
\hline mediaplay.csv & $\begin{array}{l}\text { user ID, time, album name, artist, track, track title, track } \\
\text { location, player state, track duration. }\end{array}$ \\
\hline media.csv & $\begin{array}{l}\text { user ID, record time, media file time, anonymized media } \\
\text { file name, file size. }\end{array}$ \\
\hline & \\
\hline & \\
\hline & $\begin{array}{l}\text { level, charging state, free drive space, elapsed inactive time, } \\
\text { ringing type (normal, ascending, etc.), free RAM amount. }\end{array}$ \\
\hline wlan.csv & er ID, time, first 3 bytes of MAC address, anonymized \\
\hline & $\begin{array}{l}\text { MAC address of WLAN device, anonymized SSID, signal } \\
\text { level, channel, encryption type, operational mode. }\end{array}$ \\
\hline
\end{tabular}

Table 2: Common data types of Open and Dedicated Tracks (in alphabetical order).

\begin{tabular}{|l|l|}
\hline data type & fields \\
\hline wlan_loc.csv & $\begin{array}{l}\text { user ID, time, first } 3 \text { bytes of MAC address, anonymized } \\
\text { MAC address, longitude, latitude. }\end{array}$ \\
gps.csv & $\begin{array}{l}\text { user ID, record time, time from GPS satellite, geo-location } \\
\text { (altitude, longitude, latitude), speed, heading, accuracy } \\
\text { and DOP, time since GPS system started. }\end{array}$ \\
\hline
\end{tabular}

Table 3: Specific data types for the Open Track. 


\subsection{Data anonymization}

Various anonymization techniques were applied to the MDC data: truncation for location data, and hashing of phone numbers, names (such as contacts, WLAN network identifiers, Bluetooth device identifiers), and MAC addresses. This process is summarized in this subsection.

\subsubsection{Anonymizing location data}

The detailed locations can indirectly provide personally identifiable information, therefore risking compromising the privacy of the LDCC participants. A location that is regularly used at night, for instance, could indicate the participant's address, which could then potentially be reversed using public directories to find out the participant's identity. While all researchers participating in the MDC committed in writing to respect the privacy of the LDCC participants, i.e. not trying to reverse-engineer any private data (see Section 3), we also took specific measures in terms of data processing.

Anonymizing location data for Open Track. In order to provide enough privacy protection while simultaneously keeping the data useful, we applied k-anonymity by truncating the location data (longitude, latitude) so that the resulting location rectangle, or anonymity-rectangle, contains enough inhabitants. That is, the exact location, consisting of longitude and latitude information, was replaced by a rectangular area of varying size, subsequently increasing the uncertainty of the given location. For instance, in city centers anonymity-rectangles tend to be small, while in rural areas anonymity-rectangles can be kilometers wide. This step required a considerable amount of manual work that included visualizing the most visited places of the LDCC participants in order to correctly set the size of the anonymity-rectangles. Once set, those anonymity-rectangles were applied to all data from all users.

The data for the Open Track included also the WLAN based location information which was passed through a similar anonymity-rectangle filtering process.

Location data for Dedicated Track. As discussed earlier, geo-location data was not used for the Dedicated Track. Visited places were represented by IDs which are positive integers, intrinsically removing all personally identifiable information. The mobility history of a given user is then represented as a sequence of place visits, characterizing by place ID and arrival/leaving timestamps. For the semantic place prediction task, place categories were provided for a small subset of the discovered places. We used the following categories: home; home of a friend, relative or colleague; workplace/school; place related to transportation; workplace/school of a friend, relative or colleague; place for outdoor sports; place for indoor sports; restaurant or bar; shop or shopping center; holiday resort or vacation spot.

\subsubsection{Anonymizing $M A C$ addresses, phone numbers, and text entries}

Hashing was applied to a variety of text entries appearing in the MDC data, including Bluetooth names, WLAN network identifiers (SSID), calendar titles and event locations, first names and last names in the contact lists, and media filenames (such as pictures).

For anonymization of the WLAN and Bluetooth MAC addresses, we split them into two parts. First, the MAC prefix, also known as the "Organizationally Unique Identifier (OUI)" [33], was kept in clear text. Second, the rest of the MAC address was anonymized by hashing, after concatenating it with secret key, and the userID for dedicated challenges. 


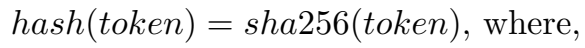

token $=($ seckey $1 \|$ information $\|$ seckey 2$)$, for open challenges,

token $=($ userID $\|$ seckey $1 \|$ in formation $\|$ seckey 2$)$, for dedicated challenges.

Note that, for the dedicated challenges, this anonymization method results in the same MAC address appearing differently in different user data sets.

Phone numbers appearing in the call logs and contact lists were also split in two parts. First, the number prefix, which contains the country and region/mobile operator codes, was left as clear text. Then, the rest of the phone number was hashed as described above. The cell ID and the location area code (LAC) of the cellular networks were also anonymized using the hashing technique described above.

\subsection{Watermarking}

The release of the MDC data set to a large community of researchers motivated an additional step in which each distributed copy of the data set was watermarked individually in order to identify it if necessary. The watermarking process introduced negligible alterations of the data that did not interfere with the results.

\section{MDC schedule and participation}

The MDC process started in summer 2011. We targeted to organize the MDC Workshop, where methods and results would be presented, within one year. We decided to keep the challenge open for all researchers with purely academic affiliation. The prospective participants of the Open Track had to submit a short proposal with their concrete plan, and the participants of the Dedicated Track had to agree to participate at least one task. While the MDC was by nature open, a series of steps was established for participant registration. Importantly, this included signature of a Terms and Conditions agreement, in which each researcher explicitly committed to use the data only for research purposes, and to treat the data in an ethical and privacy-preserving manner (reverse engineering of any portion of the MDC data to infer sensitive personal information was strictly forbidden).

The MDC registration process was launched in early November 2011 and closed in mid-December 2011. The challenge was received enthusiastically by the research community. In early January 2012, the MDC data was released to more than 500 individual participants as individually watermarked copies for more than 400 challenge tasks. The participants were affiliated with hundreds of different universities and research institutes, with a worldwide geographic distribution (Asia 23\%, USA 22\%, Europe 51\%, other regions $4 \%$ ). Many leading universities in the field participated in the MDC tracks.

A total of 108 challenge submissions were received on mid-April 2012, corresponding to 59 entries for the Dedicated Track and 49 entries for the Open Track. All submitted contributions were evaluated by a Technical Program Committee (TPC), composed of senior members of the mobile and pervasive computing communities. The TPC members did not participate in the MDC themselves to minimize possible conflicts of interest.

The criteria to evaluate entries for each Track were different. On one hand, the Open Track entries were evaluated according to a set of standard scientific criteria, including the novelty and quality of each contribution, and the paper presentation. All entries in the Open Track were reviewed at least by two members of the TPC. On the other hand, all 
entries in the Dedicated Track were evaluated using the objective performance as the only criterion to decide on acceptance to the MDC Workshop. Entries for all three dedicated tasks were compared against standard baseline methods. In addition, all Dedicated Track papers were subject to review in order to verify basic principles of originality, technical novelty, experimental correctness, and clarity. Papers corresponding to entries whose performance did not outperform the baseline were reviewed by one member of the TPC. All other papers were reviewed at least by two members. The TPC evaluated all papers without knowledge of the performance obtained on the test set. While the reviews did not play any role in the acceptance decision for the Dedicated Track, they helped to detect a few problems, and in every case they were passed on to the authors. In particular, the reviews served as guidelines to the authors of accepted entries to improve the presentation of their approach and achieved results. Final acceptance for all entries was decided during a face-to-face meeting involving all MDC co-chairs, in which all papers were discussed and in some cases additional reviews were performed. In some cases, a shepherd was assigned to accepted entries to ensure that the key comments from the reviewers were implemented. As a result of the reviewing process, 22 entries to the Open Track and 18 entries to the Dedicated Track were accepted. For the Dedicated Track, we decided not to reveal the teams' absolute performance scores and relative ranking before the MDC Workshop. Finally, a number of awards was given to the top contributions, based on the entries' performance for the Dedicated Track, and following the recommendations of an Award Committee specifically appointed for the Open Track.

\section{Analysis of the MDC submissions}

We were positively surprised by the diversity of ideas and technical approaches submitted to the Challenge. It was clear that many teams could produce promising results and original approaches in a couple of months of work with the data. With two objectives in mind (drawing a thematic map of the interests of the MDC community participating in the Open Track, and objectively comparing the performance of the methods addressing tasks of the Dedicated Track) we present an analysis of the MDC accepted submissions. We start with an analysis of the Open Track, and continue with a discussion about the Dedicated Track.

\subsection{Open Track: Diversity in Mobile Big Data Research}

The papers related to the MDC Open Track exhibited a wide range of themes, ranging from visualization techniques to behavioural analysis to the practical application of networking and connectivity technologies. This demonstrates broad opportunities related to rich mobile data. Despite of the diversity in terms of research questions and topics explored, several commonalities emerged. Many papers underlined that the MDC dataset provides an unique opportunity to validate a given model or research question. For instance, Schulz et al. [34] utilized the co-existence of cell ID and GPS traces to study the potential of the former in deriving human mobility patterns. Another recurrent theme was the utilization of multiple data modalities; for instance, De Domenico et al. [35] analyzed the correlation between spatial and social patterns. Finally, the applied nature of several papers is worth noting. Consider, e.g., Frank et al. [36], whose method can inspire the design of novel interfaces for contextual services, or the paper by McGrath 
et al. [37], whose method could constitute an alternative positioning method for cheap feature phones, which lack GPS.

A total of 21 papers were accepted to be presented in the MDC workshop. The analysis of the papers led to three main categories: Big Data at Meta Level, Behavioural Analysis, and Networking \& Connectivity. Separate sections will be devoted to describe each of these themes.

\subsubsection{Big Data at Meta Level}

The papers in this category reflected high-level Big Data issues. Several were associated with techniques for summarizing Big Data, e.g. from a visual perspective. A few entries were related to inspection and comparison of Big Data analysis techniques.

Summarizing Big Data. Hoferlin et al. [38] and Slingsby et al. [39] focused on systems that may be used for visualizing complex data sets. The former aimed to provide a tool for open-ended, exploratory analysis, through the provisioning of complementing views on the data. The authors underlined the importance of privacy-related considerations when it comes to generation of visual summaries of behaviour of individual participants. The latter paper, on the other hand, was focused on visualizing the spatial and temporal nature of social networks. This entry received the third price in the Open Track. The MDC data set was shown to have some potential but importantly, the authors also called for more comprehensive data sets, capturing more than just mobile phone based communication. Skupin and Miller [40] built on methods related to traditional geographic atlases by presenting base map configurations on top of which thematic elements, such as demographic aspects of commuting modalities of MDC participants were overlaid. The method is a proof-of-concept regarding visualization of high dimensional attribute spaces in an intuitive way. The above papers are related to visualization. Frank et al. [36] described the process of translating contextual data collected through mobile phones to natural language sentences related to locations encountered by the individuals in the campaign. The applied value of the method can be easily seen: natural language techniques could be suitable for life logging services. This work was awarded the second Open Track price.

Improving the Analysis of Big Data. The geographic modalities in the MDC data set pertain both to GPS as well as GSM traces. Schulz et al. [34] utilized the co-existence of these two modalities to study the effectiveness of GSM mobility traces in deriving human mobility patterns. GSM data was shown to be associated with weaknesses and strengths. Idrissov and Nascimento [41] used MDC data to show the quality of their trajectory clustering method. Moving from large amounts of data to more simple representations, Hartmann et al. [42], on the other hand, aimed at reducing the number of states in user traces. With the increasing popularity of location based services, both papers can have practical value.

\subsubsection{Behavioural Analysis}

The papers belonging to this category were related to analysis of spatial and social behaviors. Some of the papers were also associated with contextual dependencies in mobile phone usage behaviour.

The entries by Niinimaki et al. [43], and Gustarini and Wac [44] bear relevance to psychological research. The former paper investigated if weather has an impact on mobility patterns of individuals. Two data sets were combined - the MDC data by Nokia 
and meteorological data by MeteoSwiss. The latter paper tapped into the perception of intimacy across various locations. Bluetooth encounters, ring tone status of the participants as well as charging behavior were used as indicators of social density, type of social connections in one's surroundings, as well as perceived level of safeness in regard to a given location.

Two MDC entries were associated with examining the overlap between spatial and social behaviors. De Domenico et al. [35] showed that user movement forecasting can be improved through exploiting the correlation between movements of friends and acquaintances, an idea that was awarded the first price in the Open Track. Munjal et al. [45], on the other hand, were interested in using the correlation between locations visited by users who were socially interacting in making efficient routing decisions in mobile networks.

Muhammad and Van Laerhoeven [46], and McGrath et al. [37] were concerned with inferring social groups and locations, respectively, using indirect measures. The former paper found consistent results across a number of modalities, including shared contacts, shared IDs on the call lists, common WLAN MAC addresses seen by the users, and GPS clusters in close proximity in time and space to one another. The entry by McGrath et al., on the other hand, showed that the transitions between spatial habitats of individuals was predictable solely from daily routines and their smart phone usage habits.

McInerney et al. [47], Barmpounakis and Wac [48], and Tan et al. [49] focused on understanding mobile phone usage in different contexts. McInerney et al. established a connection between the instantaneous entropy of individuals, i.e., a measure of their momentary predictability, and the use of the mobile phone. In particular, almost all mobile applications showed an increased level of use when the individual behaves in a non-routine based way. Barmpounakis and Wac [48], on the other hand, investigated connectivity and application use patterns, with the aim of improving Quality of Service. Finally, Tan et al. [49] adopted an applied perspective through the investigation of a method capable of real-time application usage predictions in a mobile context.

\subsubsection{Networking and Connectivity}

In several cases, the MDC dataset was utilized from the networking and connectivity perspective. Keller et al. [50] studied ad hoc file sharing over WiFi among individuals with similar musical taste. Also Wu et al. [51] also investigated opportunistic data transfer. Their focus was on the use of smart phones to collect data from wireless sensor nodes. Both papers yielded positive results based on data stemming from the MDC.

Van Syckel et al. [52] set out to study information dissemination in a delay tolerant network. In low-density delay tolerant networks, the consistency in everyday user movement was found to contribute significantly to the information distribution rate.

The entry by Michaelis et al. [53] was concerned with using MDC data to predict the network cell IDs of a moving user, for the purposes of active load balancing. The generated paths of MDC participants were used in order to predict the next cell. For some participants, the experiments exceeded $80 \%$ accuracy.

Wang et al. [54] investigated periodicity of encounter patterns between mobile devices in order to improve communications in a mobile network setting. Strong weekly and daily patterns emerged; the authors also showed how the persistence of such patterns got interrupted from time to time. 


\subsection{Dedicated Track, Task 1: Semantic Place Prediction}

As stated earlier in this paper, the first task was the inference of the semantic labels of the most significant places that a user visits. This task was a multi-class classification problem, but the training and testing sets were not distributed uniformly as Figure 4 shows. As only the most visited places were labeled by the participants, it is quite natural that homes and workplaces are the most represented classes in the dataset. There were only small discrepancies between the training and test sets, but still guaranteeing enough representativeness in each class. Manual place labeling was a tedious task, and so to keep the workload reasonable, only a small fraction of them (around 10\%) was shown to the participants for labeling. Therefore, the training and test sets contained a large number of unlabeled places.

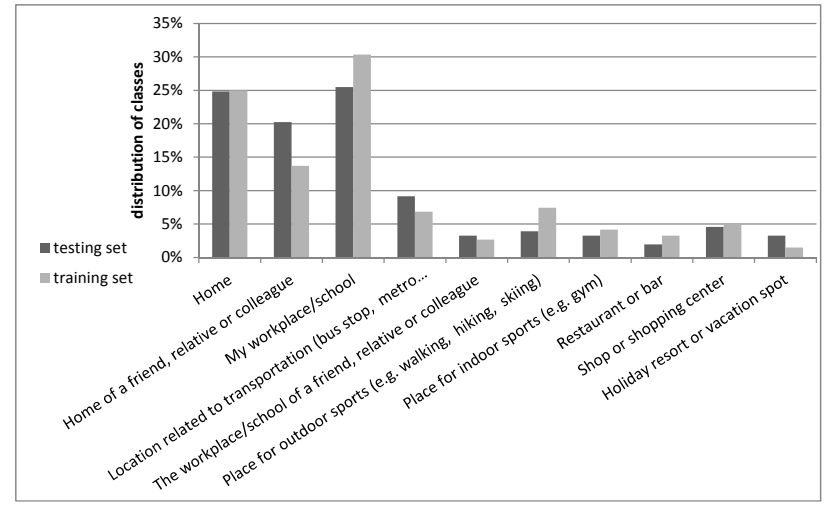

Figure 4: Distribution of the classes in the training and testing sets for the dedicated task 1.

To get a first idea of the performance of the different submitted algorithms and to allow a pre-selection at submission, a baseline classification method was implemented. Using only time features and a naive Bayes classifier, the baseline achieved $53 \%$ of accuracy. From the submissions, the most popular features were based on time and frequency of visits, but also on accelerometer features. Many teams did also decompose the problem into several binary classification problems.

The accuracy was computed as the number of correct predictions over the places in the test set that were actually labeled. The other unlabeled places were just ignored.

According to this measure, all submissions below the baseline were attributed one reviewer and rejected by default, and the other ones had at least two reviews. Figure 5 shows the distribution of the best submission for each team. As stated earlier, each team was allowed to submit blindly 5 results and the best one of them was kept for the ranking. Detailed analysis showed that the scores of the different submissions originating from the same team had a low variance and thus most likely resulted from tuning algorithm parameters rather than from generating random results.

Among the three best results, various methods were proposed and experimented.

In third position, Montoliu et al. [55] used "smart" binary classifiers using 1-vs-1 and 1-vs-2 classes. The features that were selected most often were based on time, phone 


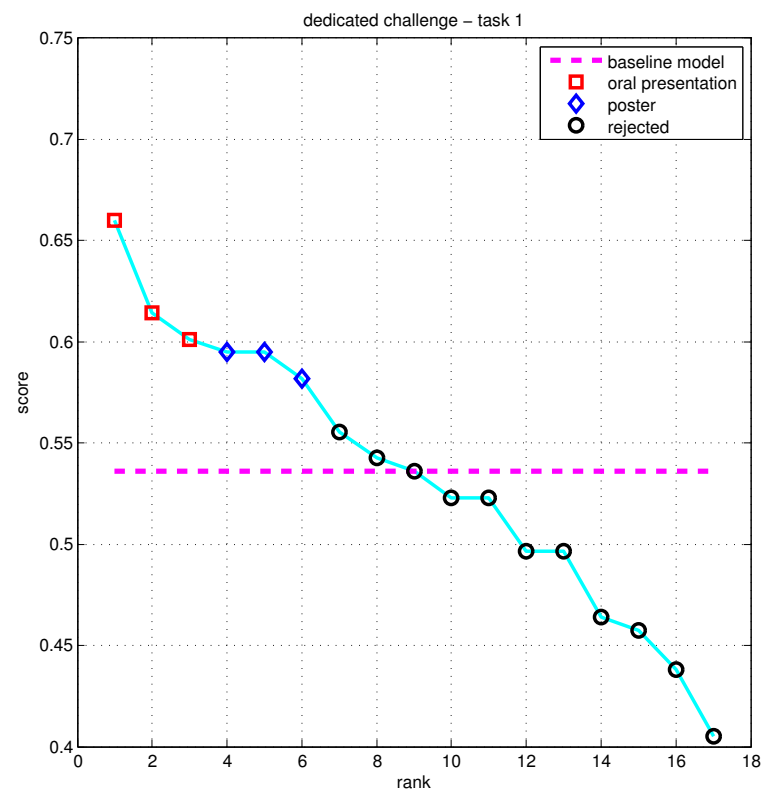

Figure 5: Final result of Dedicated Task 1. Scores of the 17 best submissions, one per team.

profile, call-logs, SMS and WLAN. Moreover, they showed good performance of a novel MultiCoded class-based method as evaluation rule for the binary classifiers.

In second position, Huang et al. [56] proposed to use a multi-level classification where the decision tree was manually built to deal with the class imbalance and included some common sense knowledge. For each level of the tree, several classifiers were trained and the features selected using $\chi^{2}$ were the following: accelerometer movements, missed-call, text-out, Bluetooth, and time of visit.

Finally in first position, Zhu et al. [57] focused on generating as many features as possible (over two million), letting the feature selection algorithms do their job. They also conditioned the features by time intervals of 30 minutes and showed a great improvement of accuracy. The most useful features were based on time, Bluetooth and accelerometer. Several classifiers like Logistic regression, SVM, Gradient Boosting Trees, and Random Forest were evaluated.

It was interesting to notice that the top contributions reached a performance roughly in the same range as the one that corresponds to the aggregated percentage for the three most common categories in the dataset (see first three columns in Fig. 4.)

Overall, this task highlighted the great challenge of giving sense and semantic meaning to collected data, based purely on mobile data inputs that do not contain explicit geolocation. The promising result obtained by the top contribution ( $66 \%$ accuracy) shows that this path is worth of more exploration. 


\subsection{Dedicated Track, Task 2: Next Place Prediction}

The goal of this task was to predict the next destination of a user given the current context, by building user-specific models that learn from the mobility history, and then by applying these models to the current context to predict where the users will go next.

For the Dedicated Track, the raw location data was transformed into sequences of place visits and the prediction task was defined in the symbolic space of place IDs. While the default minimum stay duration of visit was 10 minutes (see Section 5), we exceptionally used a larger threshold for the next place prediction task. The motivation was to filter out short visits which are very challenging to predict. In Task 2 , visits of less than 20 minutes were removed, and all evaluations were done with the sequence of visits of at least 20 minutes. The prediction context consisted of the current place ID, the arrival/leaving timestamps of the visit, and the data recorded during the visit, and the 10 minutes before arrival. The output to be predicted was the ID of the next destination.

In the training phase, the sequence of visits to places and several types of phone data associated with these visits were made available. Furthermore, in the testing phase, previously unseen data from the same set of users was provided. For each user, a number of test data points were selected randomly from unseen transitions (e.g., transition from current place to the next place) with the restriction that there was at most one test data point per day. As described above, each test data point is associated with a time interval (the mobile phone data in the test set is only available within these time intervals), and the ground truth is the next place the user visited after the time interval. As people keep visiting new places over time, both the current place $\mathrm{P}$ and the next destination $\mathrm{D}$ can be a new place that did not occur in the training set. In the ground truth, all new places that did not occur in the training set were processed by setting ID $=0$ by convention (this special category occupies $8 \%$ of the ground truth data). Classification accuracy was used as performance evaluation measure.

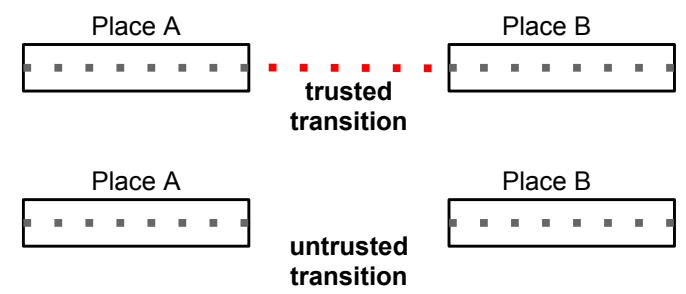

Figure 6: Trusted transition and untrusted transition. Each dot represents available location data.

Due to the missing location data during the recording process, some actual visits might have not been detected, and some detected visits might be erroneous. In other words, the next place in the recorded sequence of visits might not be the actual next place. For this reason, we introduced the concept of trusted transition, which is trusted if there are location data points of the user every 10 minutes between the leaving time of the current visit and the arrival time of the next visit, as illustrated in Figure 6. By construction, next places of trusted transitions correspond to actual next places. In the data, we found that $57 \%$ of transitions are trusted, and this indicator of trustworthiness is also provided in the preprocessed location traces. Finally, we only considered trusted transitions in the 
selection of test data points.

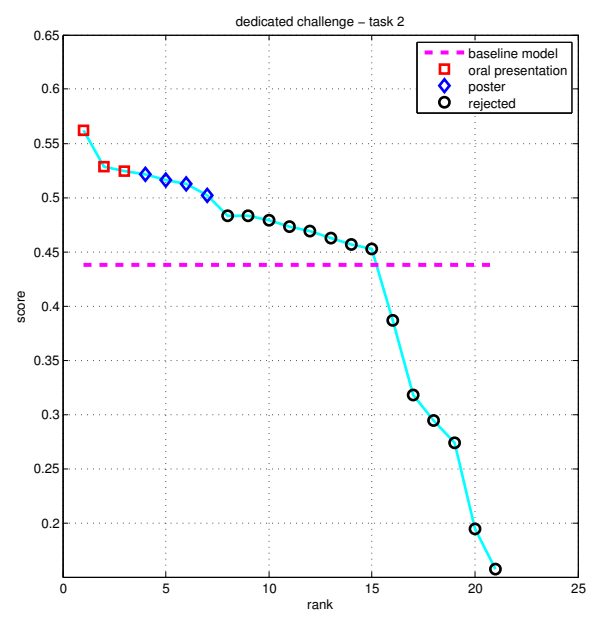

Figure 7: Final result of dedicated task 2. Accuracies of the 21 best submissions, one per team.

We received 21 valid submissions, in which $52 \%$ of the methods were reported to use only spatial and temporal context (i.e., using only mobility data). While $71 \%$ of the submissions had proposed at least one dedicated probabilistic model for user mobility, the remaining $29 \%$ of the submissions employed only standard machine learning methods such as SVM or Random Forest. The final accuracy of submissions is shown in Figure 7 in descending order, where the top 3 submissions were selected for oral presentation, and the next 4 submissions were selected for poster presentation at the MDC workshop. Note that each team had the right to submit 5 final prediction output files, and the best result was selected for each team. As a baseline method, we considered a simple method that predicted the most frequently visited place as the destination if the user is not currently at that place. If the current place is the most visited place, then the baseline method predicted the second most frequently visited place as the destination. This baseline method results in $44 \%$ of accuracy on the test set. At the third place, Gao et al [58] reached an accuracy of $52 \%$ by using a probabilistic framework that combines spatial historical trajectories with temporal periodic patterns. Wang and Prabhala [59] reached second best performance after investigating a periodicity model and SVM classifiers working on time and location features. The winner of this task reached an accuracy of $56 \%$ by combining a Dynamical Bayesian Network with two standard methods: Artificial Neural Network and Gradient Boosted Decision Trees [60].

The final result shows that human mobility is relatively difficult to predict in the considered setting. One key challenge is to learn from limited number of observations and to predict with limited contextual data. While many phone data types were provided, we found that the top 3 submissions only considered mobility data for predicting human mobility. Our interpretation is that besides spatio-temporal context, other contextual cues may be too weak for learning a human mobility model from each user data separately. 
We could expect that these additional contextual cues can be exploited more efficiently by combining generic human mobility patterns with individual mobility patterns.

\subsection{Dedicated Track, Task 3: Demographic Attribute Prediction}

Many mobile applications could benefit from being able to adapt their behavior according to the type of user. This could enable the application to provide more appropriate services or content to the user and adapt the way information is presented to the user. A straightforward way for an application to acquire information about users is to ask them to specify attributes about themselves to the application. This however requires extra effort from the user and the possibility to integrate the request of such information into the usage flow of the application. These alternatives may not always be possible, so that settings related to the user may remain unanswered. An interesting question is whether applications could infer some of the user's characteristics based on the contextual information traces that they can observe in order to facilitate appropriate adaptation of their behavior.

To investigate this question, Task 3 of the Dedicated Track was to develop methods for predicting certain demographic attributes of the users in the MDC dataset based on the context traces provided for the users.

The demographic attributes in the dataset were self-reported by the participants and covered the gender, age group, marital status, and job type of the user as well as the number of persons living in the user's household. Apart from the binary gender attribute (female and male), the age groups were modeled by binning the ages of the participants aged 16-44 years into bins of 5-6 years and providing one bin for persons older than 44 years. Marital status was reported as one of three classes: "single or divorced", "in a relationship", or, "married or living together with my partner". The job type involved four possible classes including "Training", "PhD student", "Employee without executive functions", and, "Employee exercising executive functions". Finally, the number of persons living in the user's household was modeled by five dedicated bins for one, two, three, four, and more than four persons.

The three prediction subtasks for the gender, marital status, and job type attributes were formulated as classification problems, for which the prediction accuracy was used as the evaluation measure. The prediction subtasks for age group and number of persons in the household were treated as regression problems, and the root mean squared error (RMSE) was used as the evaluation measure.

The training set consisted of context data traces from 80 distinct users, covering altogether 20492 user days. The demographic labels for users in this dataset were provided as part of the challenge data. The testing dataset contained data from 34 users, covering altogether 11606 user days. The demographic labels for users in the testing dataset were not revealed to the challenge participants. The ranking of the challenge contributions was based on the relative improvement that the submitted prediction results provided over the results obtained from very basic, dummy prediction models. The dummy prediction model used for the classification subtasks was such that it always predicted the class with the highest number of occurrences in the training data. For the regression subtasks, the dummy model always predicted the average of the attribute values in the training data.

The basic evaluation measure for classification subtasks is the overall error rate, that is the fraction of classifications made that were incorrect. For the regression problems, 
root mean square error (RMSE) is used as the basic evaluation measure. The evaluation score for each subtask was then calculated as the relative improvement of the submitted prediction over the dummy prediction. The final evaluation score for each challenge submission was then determined by taking the average of evaluation scores of the five prediction subtasks.

In addition to the evaluation, we also applied straightforward baseline prediction methods to obtain a reference against which to compare the performance of the submissions. For this, only the mobility-related features in the dataset were used. The baseline model used naive Bayesian inference for the classification subtasks and linear regression for the regression subtasks. The performance of the baseline model in comparison to the submissions for the MDC can be seen in Figure 8.

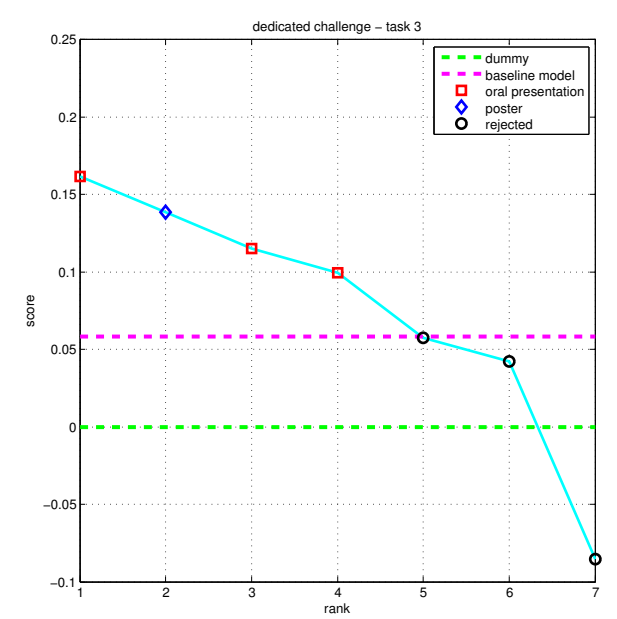

Figure 8: Final result of Dedicated Task 3. Scores of the best submissions, one per team.

As third in the final MDC ranking Brdar, Culibrk and Crnojevic [61] approached the task by extracting forty different features from the dataset and experimenting with models based on k-nearest neighbor $(\mathrm{kNN})$ graphs, mutual $\mathrm{kNN}$ graphs, radial basis function networks $(\mathrm{RBFN})$, and random forests. The $\mathrm{kNN}$ graph-based prediction model was applied both on the full feature set, and a limited feature set determined by a feature selection. For the gender prediction subtask, Brdar et al. obtained best results on the training data by using the Random Forest. For the age group, marital status, and job type subtasks, kNN with feature selection provided the best performance, whereas for the number of persons in the household subtask, both RBNF and Random Forest provided good results.

The runners-up for this dedicated task, Mohrehkesh, Ji, Nadeem and Weigle [62], generated 1100 raw features from the input data and applied feature selection methods. They then applied support vector machines (SVM) and random forests in their prediction models.

The winners of the demographic attribute prediction task, Mo, Tan, Zhong and Yang 
[63] , used an approach in which they constructed a set of tens of thousands of raw features representing conditional probabilities of user actions and applied feature filtering and dimensionality reduction to the feature set. The prediction models utilized included C4.5, gradient boosted tree, random forest, SVM, logistic regression, RepTree, support vector regression, Gaussian process, linear regression, and lasso.

\section{Discussion}

Nine months after the conclusion of the Challenge, we are in a position to reflect upon our initiative. This section provides a retrospective discussion on our motivations, design choices, issues regarding the concrete implementation of the challenge, and the main outcomes and limitations of the initiative.

\subsection{Motivations to implement $M D C$}

As described in earlier sections of this paper, before the MDC there was already a significant investment behind the LDCC data collection on a level that is obviously beyond the capabilities of individual researchers or many research groups. The data collection had initially a two-fold motivation. Such rich data was needed for research within the groups behind this initiative and among their closest partners. But from the very beginning the intention was also to share this data with research community. Therefore, the campaign was also designed so that it was possible to address rather different research questions using the same multi-dimensional data set.

The wide sharing of this data asset was motivated by the spirit of open innovation, i.e. by thinking that sharing the data was a key to scale up the overall innovation process around it. This also extended the coverage of innovation to research questions which did not match the focus and competence profile of the research groups behind the LDCC and MDC. Therefore, our intention was to enable scientific advances on different disciplines. Open data sharing brought along many research teams which had needed competencies, but without access to advanced mobile data sets before the MDC.

From the research perspective, it was liberating and empowering to enable a research community by opening the data set for wider use. Before the MDC we regularly received questions and requests regarding sharing of the LDCC data. However, it was necessary to start the data usage in a smaller scale, and the extension of the community became possible only after previous knowledge of this particular data set had accumulated.

\section{2. $M D C$ design choices}

Perhaps the key design choice was the decision to allow both open entries and specific entries. The overall result of this was a significant participation on both fronts. The participation profile also shows that the Open and Dedicated Tracks attracted different communities. The tasks chosen in the Dedicated Track proved to be challenging enough (which demonstrates the need for further research in this domain), while at the same time attractive enough to motivate researchers to test advanced data mining methods. The tasks in this Track were also diverse enough to generate interest either as individual tasks or collectively (for those teams who could target more than one). We anticipate that the definition of these tasks will result in future publications where improvements over the 
best performance obtained so far will be reported. Finally, the dual Open/Dedicated format resulted in a considerable additional effort for us as organizers, as a fully automated way of assessing the entries was no longer possible.

The decision of having multiple tracks and tasks created the need to split the data. Obviously, this lead to trade-offs between the amount of data versus the number of separate tracks. This was also the decision that implied a significant amount of work until we achieved a satisfactory solution for splitting the overall data into sub sets with appropriate amount and quality. As shown in Figure 3 and discussed more in details in Section 5 , the data split had to satisfy multiple time and population-related constraints. After careful inspection, the data of some LDCC users was deemed not useful for any of the tasks in the Challenge and was not distributed. This was mainly caused by the sparse or incomplete device usage of these particular users. Defining a single task or track would have provided the maximum amount of data but would have reduced participation.

Another design choice (in this case of LDCC rather than of MDC) was the population that took part in the data collection initiative. As stated earlier, the LDCC population does not exactly match the overall demographics of French-speaking Switzerland. On the other hand, it corresponds well to the consumer segment of real smartphone users. This makes it attractive to investigate consumer behaviour, using this particular data from the perspective of smartphone service concepts. We have also recently demonstrated that some large-scale mobility trends observed in the MDC data match patterns obtained with an independent larger-scale population and their mobility traces (Foursquare) [64]. This proves the value of the MDC data as a snapshot of real European life, beyond the tasks we proposed.

The decision of not distributing geo-location data for the Dedicated Track was motivated by our interest in promoting the development of techniques that are locationprivacy sensitive. This was a critical factor for service concept creation by following the Privacy by Design principles [8]. On the other hand, being aware of the huge interest of the community in having access to longitudinal location traces, we decided to release them as a part of the Open Track data set, with an additional location anonymization step performed for the sensitive, personal locations. When the location data sets for the Dedicated Track were prepared, the parameters had to be carefully selected (e.g. retaining shorter or longer stays) so that the data was reliable but did not compromise the amount of extracted places.

Further decisions were made related to the type of participation and the duration of the challenge. Most participants decided to work in groups. The maximum group size was an important parameter influencing the ambition level of the proposed ideas and the quality of their final implementation. We had no way of testing this a priori, and therefore decided to target the challenge for small groups. Our intention was to encourage a tight integration of effort. The same logic was used when the duration of the Challenge was decided. Three months were considered as a period that would allow seriously involved people to achieve something significant, without resulting in a prohibitive period that would distract the participants from their main activities (counting on most of them being students or postdocs). It was also considered beneficial to execute the whole Challenge within less than a year to maintain the momentum throughout the whole initiative.

A final design issue was related to data distribution. Existing platforms, like Kaggle or Crawdad, are commonly used in research. But decided to keep the data distribution close to the organizing team, in order to control all aspects of authorization, access, 
individually watermarked copies, etc. This choice was a clear one: we owe this level of care to the volunteers in our study.

\subsection{MDC implementation}

While the above section relates to the design of MDC, we now discuss aspects related to the implementation of this initiative. The relevant themes are as follows: community formation, privacy and legal aspects, as well as outcome analysis.

Community formation. The community accessing and analyzing the data evolved in the course of the LDCC and MDC. As explained earlier, a core team was formed around the data during the LDCC stage. The network became significantly wider during the MDC stage. The core team, which was involved throughout the process and facilitated all stages, consisted of employees from NRC Lausanne and Idiap. In retrospect, we feel that the continuity and long-term commitment of this small core team was one of the most critical factors enabling the scaling up of the community. The core team had a holistic responsibility both for LDCC and MDC, covering also the practicalities related to these initiatives. Various aspects had to be mastered, including hosting of the data, establishing a legal framework for data sharing, taking care of the privacy aspects, as well as controlling the data access in regard to additional research groups joining in during the subsequent stages. The Nokia and Idiap teams were located in proximity of one another, and they operated in a very seamless manner with a lot of collocated meetings. The overall MDC schedule was designed to be tight, and during many time critical phases the physical proximity was of particular importance.

From this well functioning core team, it was rather natural to scale up the community to a larger level. Acquiring a strong familiarity with the dataset during the LDCC stage enabled critical aspects to be taken into account when starting to significantly increase the community size during the MDC.

Privacy aspects. Privacy protection required extremely careful considerations due to multimodality of the rich smartphone data. We already described the necessary countermeasures both when the smartphone data was originally collected and when it was later released to the research community. In practice, this required both technical countermeasures and agreement-based privacy protection. In that manner it was possible to achieve an appropriate balance between the necessary privacy protection, while simultaneously maintaining the richness of the data for research purposes.

When it comes to lessons learned in respect to the privacy aspects of the process, two issues are worth emphasizing. First, the workload associated with privacy protection should not be underestimated. For example, the manual location obfuscation process was time-consuming and had to be split among all members of the core team. Second, we learned that despite serious attempts in adhering to the privacy protection principles, some of the research findings can nevertheless be associated with privacy issues. For example, when reviewing the challenge entries, some of the submissions had conducted analysis at the level of individuals rather than at population level. Therefore, the core team has an important role to play not only when providing access to the data, but also when inspecting the outcomes of the analysis across the various participating teams. Implementation of a privacy review process in regard to publications based on the mobility data is highly recommended to ensure maximal elimination of unintentional and accidental privacy issues. 
In addition to implementing this type of privacy review, we also learned that a specific philosophy is needed when the responsibility that the core team has adopted toward the data collection participants is transferred to the extended network of MDC researchers. All data users need to have an understanding of what is acceptable and they need to be responsible and respect the underlying principles. Therefore, the core team has an essential educative role in establishing such principles and guidelines, articulating clearly what is acceptable from the privacy point of view. It is important to use concrete examples to illustrate the principles in a clear and understandable manner. Also, the guidelines have to play a prominent role in the overall communications toward the participating teams.

Legal aspects. The collection of rich mobile data meant that severe responsibility towards the data collection campaign participants had been taken. Therefore, an appropriate legal framework had to be established to transfer this responsibility to the researchers using the shared data. In our context, several data sharing frameworks were prepared: one for initial institutional data sharing partners before the MDC, one for MDC participation, and finally one for extended use of the MDC data after the challenge itself. This all creates a significant burden of legal work which should not be underestimated when further such initiatives are planned or prepared. As a consequence of global coverage of the initiatives and different legislation in different parts of the world, some organizations came up with special wishes and modification requirements which easily leads to iterative interactions and high investment with respect to usage of legal resources. Such interactions, however, offer also one channel to communicate the related responsibilities and importance of underlying privacy matters. On the other hand, it is essential that the understanding about the responsibilities propagates among the involved researchers, not only in the legal teams of the organizations.

\subsection{Other implications}

Already so far the Mobile Data Challenge has produced interesting findings and multidisciplinary scientific advances. The contributions to the MDC addressed various interesting angles from the perspective of mobile computing research, like investigations on predictability of human behavior patterns or opportunities to share/capture data based on human mobility, visualization techniques for complex data as well as correlation between human behavior and external environmental variables, such as weather patterns.

On the other hand, the establishment of a community around this particular data set has already been an achievement as such. It is too early to make a final assessment regarding the overall scientific findings less than a year after the MDC. Many of the participating teams have continued their efforts, and extended outcomes and complete new research tracks will be reported in the open literature during the months and years to come.

However, one of the observations already at this point is that the range of topics associated with the submissions was surprisingly wide and multidisciplinary, consequently imposing challenges on the review process. While the dedicated tasks were quite strictly associated with using machine learning techniques, the open challenge entries varied widely. The reviewers needed, therefore, to assess a range of disciplines, from computer science to social sciences, from visualization and HCI to geography. This requires multidisciplinary review teams to ensure a fair and reliable review process independent of the scientific angle of the analysis. 
We also noticed that most of the research was conducted at a theoretical level. An applied angle, especially in regard to user experience aspects, was less frequently encountered. More specifically, not many challenge entries took a stance on what would be the manifestation of a given pattern based on mobility data, in terms of concrete application or service running on the mobile phone, and bringing concrete benefits to the end users. One way of supporting such applied, UX centric angle in the future would be to extend the challenge more explicitly to design and HCI communities.

\section{Conclusions}

This paper described a systematic flow of research over four years, targeting to create and provide unique longitudinal smartphone data for wide use by the research community. In this paper we motivated our initiative and summarized the key aspects of the Lausanne Data Collection Campaign (LDCC) in which the rich smartphone data was collected from around 200 individuals over more than a year. We also described in further details the Mobile Data Challenge (MDC) by Nokia, which was a data analytics contest making this data widely available to the research community. The data collection campaign run in 2009-2011 whereas the challenge was organized in 2011-2012.

Collecting such data requires extensive effort and underlying investments, which often means that data sets are available for researchers only in the limited manner. This has recently generated discussions about the basic principles of science in connection with Big Data driven research. Verification of claimed scientific findings can be challenging if access to data is limited. Protecting privacy of individuals behind the data is obviously the key reason for access and usage limitations of Big Data.

We demonstrated that data sharing with the research community and open innovation momentum around common resource are both possible. Achieving that required a holistic approach on privacy throughout the whole flow of design and execution of the LDCC and MDC initiatives. Privacy protection requires extremely careful considerations. In this paper we described the necessary countermeasures both when the smartphone data was originally collected and when it was later released to the research community. In practice this required both technical countermeasures and agreement based privacy protection.

Running the LDCC and MDC required significant long-term commitment from the teams behind these initiatives. This commitment was a key to build extended initiatives for data sharing by utilizing the knowledge accumulated over several years. Furthermore, the commitment related to this data and the community established around it remains strong. In practice, the dataset continues to be accessible both for MDC participants and new users through a Data Sharing Agreement framework [65] (managed by Idiap since early 2013). Based on our experience, if something similar is planned by other research organizations in the future, the overall effort required should not be underestimated in the planning phase, and the long-term commitment of the organizing teams needs to be ensured. Otherwise, the quality level of execution might be risked, which might have undesired impact e.g. on privacy aspects.

If we started organizing the MDC initiative again, based on our experience today, we would to a large extent repeat the same design choices and other decisions. But if we started to design a successor initiative for the MDC now, taking into account the existing community and past history, the expansion of the community towards new disciplines would be an interesting option. In practice, this would be possible by scoping the new 
challenge increasingly towards the HCI community. An Open Track format together with the fresh angle of the new community could potentially lead to radically new innovations around the same data.

Already so far the Mobile Data Challenge has produced interesting findings and multidisciplinary scientific advances. The contributions to the MDC addressed many angles in mobile computing. The materials presented in the MDC workshop are available in [11]. Obviously the established community around this data will continue producing novel findings. Therefore, we plan to maintain an updated list of the most important LDCC and MDC research outcomes in the future. The initiatives described in this paper create a solid basis for future innovation. This is not only because of open data sharing, but also because the MDC provided a set of benchmarks with accurate documentation.

Finally, the momentum around MDC is expected to continue and expand. The MDC resources remain valid to analyze various research questions in the future, even though the data was originally collected in 2009-2011. Many of the underlying behavioral patterns captured by the data do not change quickly; Only the details related to application usage might be more device-specific, and therefore outdate in a shorter time. The contest format of the MDC limited the interactions among the participating teams during the contest itself. On the other hand, now when the community continues their research around the same data, a community spirit is highly encouraged. In practice, this can mean early and open sharing of the research findings and shared tools for data processing and visualization. We could facilitate that kind of momentum by arranging the needed channels.

In conclusion, we look forward to seeing further innovations from the community working together.

\section{Acknowledgments}

We sincerely thank all the volunteers in the LDCC initiative for their participation and contributed data, and all the researchers who responded to our open invitation to sign up and participate in the MDC. We also thank Niko Kiukkonen for his key role with the arrangements of the Lausanne Data Collection Campaign. David Racz's pioneering work with the Nokoscope and Simple Context data collection systems is also gratefully acknowledged. Additionally we thank Emma Dorée, Antti Rouhesmaa, and various other people from Nokia for their contributions to the arrangements of the Mobile Data Challenge.

\section{References}

[1] P. Ross, Top 11 technologies of the decade, Spectrum, IEEE 48 (2011) 27-63.

[2] N. Eagle, A. Pentland, D. Lazer, Inferring friendship network structure by using mobile phone data, Proceedings of the National Academy of Sciences 106 (2009) 15274-15278.

[3] M. C. Gonzalez, C. A. Hidalgo, A.-L. Barabasi, Understanding individual human mobility patterns, Nature 453 (2008) 779-782.

[4] G. Chittaranjan, J. Blom, D. Gatica-Perez, Mining large-scale smartphone data for personality studies, Personal and Ubiquitous Computing 17 (2013) 433-450.

[5] S. Isaacman, R. Becker, R. Cáceres, S. Kobourov, M. Martonosi, J. Rowland, A. Varshavsky, Identifying important places in people's lives from cellular network data, in: Proc. Int. Conf. on Pervasive Computing, San Francisco, 2011. 
[6] L. Backstrom, E. Sun, C. Marlow, Find me if you can: improving geographical prediction with social and spatial proximity, in: Proc. World Wide Web Conf. (WWW), 2010.

[7] "http://crawdad.cs.dartmouth.edu/", 2012.

[8] "http://privacybydesign.ca/", 2012.

[9] I. Aad, V. Niemi, NRC Data Collection and the Privacy by Design Principles, in: PhoneSense, 2011.

[10] J. Laurila, D. Gatica-Perez, I. Aad, J. Blom, O. Bornet, T.-M.-T. Do, O. Dousse, J. Eberle, M. Miettinen, The mobile data challenge: Big data for mobile computing research, in: Proc. Mobile Data Challenge by Nokia Workshop, in conjunction with Int. Conf. on Pervasive Computing, Newcastle, UK, 2012.

[11] "http://research.nokia.com/mdc", 2012.

[12] R. Becker, R. Cáceres, K. Hanson, S. Isaacman, J. M. Loh, M. Martonosi, J. Rowland, S. Urbanek, A. Varshavsky, C. Volinsky, Human mobility characterization from cellular network data, Communications of the ACM 56 (2013) 74-82.

[13] V. D. Blondel, M. Esch, C. Chan, F. Clerot, P. Deville, E. Huens, F. Morlot, Z. Smoreda, C. Ziemlicki, Data for development: the d4d challenge on bile phone data, arXiv preprint arXiv:1210.0137 (2012).

[14] I. Guyon, V. Lemaire, M. Boullé, G. Dror, D. Vogel, Analysis of the kdd cup 2009: Fast scoring on a large orange customer database (2009).

[15] S. Consolvo, D. W. McDonald, T. Toscos, M. Y. Chen, J. Froehlich, B. Harrison, P. Klasnja, A. LaMarca, L. LeGrand, R. Libby, et al., Activity sensing in the wild: a field trial of ubifit garden, in: Proceedings of the SIGCHI Conference on Human Factors in Computing Systems, ACM, 2008, pp. 1797-1806.

[16] CENS/UCLA, Participatory sensing / urban sensing projects, "http://research.cens.ucla. edu/", 2012.

[17] I. Berkeley, Urban atmospheres, "http://www.urban-atmospheres.net/", 2012.

[18] J. Paek, J. Kim, R. Govindan, Energy-efficient rate-adaptive gps-based positioning for smartphones, in: Proceedings of the 8th international conference on Mobile systems, applications, and services, ACM, 2010, pp. 299-314.

[19] Y. Wang, J. Lin, M. Annavaram, Q. A. Jacobson, J. Hong, B. Krishnamachari, N. Sadeh, A framework of energy efficient mobile sensing for automatic user state recognition, in: Proceedings of the 7th international conference on Mobile systems, applications, and services, ACM, 2009, pp. $179-192$.

[20] "https://www.sensenetworks.com", 2012.

[21] U. Berkeley/Nokia/NAVTEQ, Mobile millennium, "http://traffic.berkeley.edu", 2012

[22] A. Thiagarajan, L. Ravindranath, K. LaCurts, S. Madden, H. Balakrishnan, S. Toledo, J. Eriksson, Vtrack: accurate, energy-aware road traffic delay estimation using mobile phones, in: Proceedings of the 7th ACM Conference on Embedded Networked Sensor Systems, ACM, 2009, pp. 85-98.

[23] N. Eagle, A. Pentland, Reality mining: sensing complex social systems, Personal and ubiquitous computing 10 (2006) 255-268.

[24] N. Kawaguchi, H. Watanabe, T. Yang, N. Ogawa, Y. Iwasaki, K. Kaji, T. Terada, K. Murao, H. Hada, S. Inoue, et al., Hasc2012corpus: Large scale human activity corpus and its application, http://hasc.jp/en (2012)

[25] N. Kiukkonen, J. Blom, O. Dousse, D. Gatica-Perez, J. Laurila, Towards rich mobile phone datasets: Lausanne data collection campaign, in: Proc. Int. Conf. on Pervasive Services, Berlin, 2010.

[26] R. Montoliu, D. Gatica-Perez, Discovering human places of interest from multimodal mobile phone data, in: Proc. Int. Conf. on Mobile and Ubiquitous Multimedia, Limassol, 2010.

[27] M. Przybocki, A. Martin, Nist speaker recognition evaluation-1997, in: Proceedings of RLA2C 1998, pp. $120-123$

[28] A. Smeaton, P. Over, W. Kraaij, Evaluation campaigns and trecvid, in: Proceedings of the 8th ACM international workshop on Multimedia information retrieval, ACM, 2006, pp. 321-330.

[29] J. Bennett, S. Lanning, The netflix prize, in: Proceedings of KDD Cup and Workshop, volume 2007, 2007, p. 35

[30] R. Bell, J. Bennett, Y. Koren, C. Volinsky, The million dollar programming prize, Spectrum, IEEE 46 (2009) 28-33.

[31] T. Do, D. Gatica-Perez, Contextual conditional models for smartphone-based human mobility prediction, in: Proc. ACM Int. Conf. on Ubiquitous Computing, Pittsburgh, 2012

[32] J. Blumenstock, D. Gillick, N. Eagle, Who's calling? demographics of mobile phone use in rwanda, in: Proc. AAAI Artificial Intelligence for Development, AAAI, 2010 
[33] "http://en.wikipedia.org/wiki/MAC_address", 2012.

[34] D. Schulz, S. Bothe, C. Körner, Human mobility from gsm data-a valid alternative to gps, in: Proc. Mobile Data Challenge by Nokia Workshop, in conjunction with Int. Conf. on Pervasive Computing, Newcastle, UK, 2012.

[35] M. De Domenico, A. Lima, M. Musolesi, Interdependence and predictability of human mobility and social interactions, in: Proc. Mobile Data Challenge by Nokia Workshop, in conjunction with Int. Conf. on Pervasive Computing, Newcastle, UK, 2012.

[36] J. Frank, S. Mannor, D. Precup, Generating storylines from sensor data, in: Proc. Mobile Data Challenge by Nokia Workshop, in conjunction with Int. Conf. on Pervasive Computing, Newcastle, UK, 2012.

[37] R. McGrath, C. Coffey, A. Pozdnoukhov, Habitualisation: localisation without location data, in: Proc. Mobile Data Challenge by Nokia Workshop, in conjunction with Int. Conf. on Pervasive Computing, Newcastle, UK, 2012.

[38] B. Hoferlin, M. Hoferlin, J. Rauchle, Visual analytics of mobile data, in: Proc. Mobile Data Challenge by Nokia Workshop, in conjunction with Int. Conf. on Pervasive Computing, Newcastle, UK, 2012.

[39] A. Slingsby, R. Beecham, J. Wood, Visual analysis of social networks in space and time, in: Proc. Mobile Data Challenge by Nokia Workshop, in conjunction with Int. Conf. on Pervasive Computing, Newcastle, UK, 2012.

[40] A. Skupin, H. J. Miller, Nokia MDC atlas: An exploration of mobile phone users, land cover, time, and space, in: Proc. Mobile Data Challenge by Nokia Workshop, in conjunction with Int. Conf. on Pervasive Computing, Newcastle, UK, 2012.

[41] A. Idrissov, M. A. Nascimento, A trajectory cleaning framework for trajectory clustering, in: Proc. Mobile Data Challenge by Nokia Workshop, in conjunction with Int. Conf. on Pervasive Computing, Newcastle, UK, 2012.

[42] F. Hartmann, C. P. Mayer, I. Baumgart, Mobreduce: Reducing state complexity of mobility traces, in: Proc. Mobile Data Challenge by Nokia Workshop, in conjunction with Int. Conf. on Pervasive Computing, Newcastle, UK, 2012.

[43] M. Niinimaki, T. Niemi, Where do people go when it rains?, in: Proc. Mobile Data Challenge by Nokia Workshop, in conjunction with Int. Conf. on Pervasive Computing, Newcastle, UK, 2012.

[44] M. Gustarini, K. Wac, Estimating people perception of intimacy in daily life from context data collected with their mobile phone, in: Proc. Mobile Data Challenge by Nokia Workshop, in conjunction with Int. Conf. on Pervasive Computing, Newcastle, UK, 2012.

[45] A. Munjal, T. Mota, T. Camp, Exploring social interactions via multi-modal learning, in: Proc. Mobile Data Challenge by Nokia Workshop, in conjunction with Int. Conf. on Pervasive Computing, Newcastle, UK, 2012.

[46] S. A. Muhammad, K. V. Laerhoven, Discovery of user groups within mobile data, in: Proc. Mobile Data Challenge by Nokia Workshop, in conjunction with Int. Conf. on Pervasive Computing, Newcastle, UK, 2012.

[47] J. McInerney, S. Stein, A. Rogers, N. R. Jennings, Exploring periods of low predictability in daily life mobility, in: Proc. Mobile Data Challenge by Nokia Workshop, in conjunction with Int. Conf. on Pervasive Computing, Newcastle, UK, 2012.

[48] S. Barmpounakis, K. Wac, Deriving connectivity and application usage patterns from longitudinal mobile phone usage data, in: Proc. Mobile Data Challenge by Nokia Workshop, in conjunction with Int. Conf. on Pervasive Computing, Newcastle, UK, 2012.

[49] C. Tan, Q. Liu, E. Chen, H. Xiong, Prediction for mobile application usage patterns, in: Proc. Mobile Data Challenge by Nokia Workshop, in conjunction with Int. Conf. on Pervasive Computing, Newcastle, UK, 2012.

[50] B. Keller, P. von Bergen, S. Welten, On the feasibility of opportunistic ad hoc music sharing, in: Proc. Mobile Data Challenge by Nokia Workshop, in conjunction with Int. Conf. on Pervasive Computing, Newcastle, UK, 2012.

[51] X. Wu, K. N. Brown, C. J. Sreenan, Analysis of smart phone user mobility traces for opportunistic data collection, in: Proc. Mobile Data Challenge by Nokia Workshop, in conjunction with Int. Conf. on Pervasive Computing, Newcastle, UK, 2012.

[52] S. VanSyckel, D. Schfer, G. Schiele, C. Becker, Evaluation of an epidemic information distribution scheme in mobile ad-hoc networks, in: Proc. Mobile Data Challenge by Nokia Workshop, in conjunction with Int. Conf. on Pervasive Computing, Newcastle, UK, 2012.

[53] S. Michaelis, N. Piatkowski, K. Morik, Predicting next network cell ids for moving users with discriminative and generative models, in: Proc. Mobile Data Challenge by Nokia Workshop, in 
conjunction with Int. Conf. on Pervasive Computing, Newcastle, UK, 2012.

[54] Z. Wang, M. A. Nascimento, M. MacGregor, On the analysis of periodic mobility behaviour, in: Proc. Mobile Data Challenge by Nokia Workshop, in conjunction with Int. Conf. on Pervasive Computing, Newcastle, UK, 2012.

[55] R. Montoliu, A. Martinez-Uso, J. Martinez-Sotoca, Semantic place prediction by combining smart binary classifiers, in: Proc. Mobile Data Challenge by Nokia Workshop, in conjunction with Int. Conf. on Pervasive Computing, Newcastle, UK, 2012.

[56] C.-M. Huang, J. J.-C. Ying, V. S. Tseng, Mining users behaviors and environments for semantic place prediction, in: Proc. Mobile Data Challenge by Nokia Workshop, in conjunction with Int. Conf. on Pervasive Computing, Newcastle, UK, 2012

[57] Y. Zhu, E. Zhong, B. Wu, Feature engineering for place category classification, in: Proc. Mobile Data Challenge by Nokia Workshop, in conjunction with Int. Conf. on Pervasive Computing, Newcastle, UK, 2012.

[58] H. Gao, J. Tang, H. Liu, Mobile location prediction in spatio-temporal context, in: Proc. Mobile Data Challenge by Nokia Workshop, in conjunction with Int. Conf. on Pervasive Computing, Newcastle, UK, 2012.

[59] J. Wang, B. Prabhala, Periodicity based next place prediction, in: Proc. Mobile Data Challenge by Nokia Workshop, in conjunction with Int. Conf. on Pervasive Computing, Newcastle, UK, 2012.

[60] V. Etter, M. Kafsi, E. Kazemi, Been there, done that: What your mobility traces reveal about your behavior, in: Proc. Mobile Data Challenge by Nokia Workshop, in conjunction with Int. Conf. on Pervasive Computing, Newcastle, UK, 2012.

[61] S. Brdar, D. Culibrk, V. Crnojevic, Demographic attributes prediction on the real-world mobile data, in: Proc. Mobile Data Challenge by Nokia Workshop, in conjunction with Int. Conf. on Pervasive Computing, Newcastle, UK, 2012.

[62] S. Mohrehkesh, S. Ji, T. Nadeem, M. C. Weigle, Demographic prediction of mobile user from phone usage, in: Proc. Mobile Data Challenge by Nokia Workshop, in conjunction with Int. Conf. on Pervasive Computing, Newcastle, UK, 2012.

[63] K. Mo, B. Tan, E. Zhong, Your phone understands you, in: Proc. Mobile Data Challenge by Nokia Workshop, in conjunction with Int. Conf. on Pervasive Computing, Newcastle, UK, 2012.

[64] E. Malmi, T. Do, D. Gatica-Perez, Checking in or checked in: Comparing large-scale manual and automatic location disclosure patterns, in: Proc. Int. Conf. on Mobile and Ubiquitous Multimedia, Ulm, 2012.

[65] "http://www.idiap.ch/project/mdc/", 2013. 